\title{
Wirtschaft, Wissenschaft und Politik: Die sozialwissenschaftliche Bedingtheit linker Reformpolitik
}

Das „grundlegende Problem der Gegenwart“ ist Colin Crouchs (2008) Buch Postdemokratie zufolge die „Macht der Wirtschaftseliten“. Eine Macht, die sich auf vielfältigste Weise manifestiert: als Verhandlungsmacht transnationaler Konzerne gegenüber Regierungen, wenn es um Verlagerung von Produktionsstandorten geht; als Expertenmacht wirtschaftsnaher Lobbyisten, die als „hired guns“ politische Regulierungsprozesse maßgeblich mitgestalten, und als ökonomische Macht, die über Eigentum an Medienkonzernen, Parteispenden und Anzeigen gesellschaftliche Diskurse beeinflusst.

Zu dem, was gemeinhin als „neoliberale Hegemonie“ bezeichnet wird und sich in Lohnzurückhaltung, Standortwettbewerb sowie Sozial- und Demokratieabbau manifestiert, gerinnen diese verschiedenen Machtmechanismen allerdings erst dank der neoklassischen Vormacht in den Wirtschaftswissenschaften. Die theoretisch und methodisch monistische Ökonomie ist Stichwortgeber und Metaphernlieferant neoliberaler Diskurshoheit und Schmiermittel neoliberaler Politikprojekte (Kapeller/Huber 2009, Ötsch 2009, Lübbe 2010). Wie es um Meinungsvielfalt und ideologische Ausrichtung insbesondere der deutschen Wirtschaftswissenschaften bestellt ist, lässt sich kaum besser illustrieren als an Hand des „Hamburger Appells“ des marktradikalen Arbeitgeber-Think-Tanks „Initiative Neue Soziale Marktwirtschaft“ (INSM) aus dem Jahr 2005. Dessen Überschrift lautete „250 Professoren - 10 Thesen - 1 Meinung “. ${ }^{1}$ Fünf Jahre und eine Finanzmarktkrise später präsentieren dieselben Wirtschaftswissenschaftler dieselben Rezepte wie damals, plädieren wiederum für Sozialabbau und Lohnzurückhaltung als Ausweg aus einer Krise, die nicht zuletzt durch neoklassisch inspirierte Deregulierungs- und Verteilungspolitik mit verursacht worden war.

1 Die vollständige Liste der Unterzeichner wurde inzwischen von der Homepage der INSM entfernt, einen Eindruck verschafft noch die offizielle Homepage des Hamburgischen WeltWirtschaftsInstituts, vgl. http://www.hwwi.org/uploads/tx_wilpubdb/INSM _ Hamburger_Appell10_Straubhaar.pdf 
Ein Wandel in der ökonomischen Forschung und Lehre vergleichbar dem Aufkommen des Keynesianismus in der Makroökonomie nach der Weltwirtschaftskrise in den 1930er Jahren ist zu Beginn des 21. Jahrhunderts allerdings äußerst unwahrscheinlich. Waren damals neben dem neoklassischen Paradigma auch noch institutionelle, historische und Vorläufer evolutionärer Ansätze relativ weit verbreitet, sind derart „heterodoxe "Schulen ${ }^{2}$ mittlerweile (auch: institutionell) völlig an den Rand gedrängt (vgl. Dobusch/Kapeller 2009a, 2009b): Die Zahl der Institute und ProfessorInnen, die sich zu einem alternativen oder "heterodoxen “ Paradigma bekennen, nimmt stetig ab, ${ }^{3}$ die internationale Standardisierung führt zu einem Verlust an ökonomischem Wissen mit regionaler Tradition (man denke etwa an die deutsche Ordnungsökonomik, die erst jüngst versuchte, sich mittels einer öffentlichen Petition Aufmerksamkeit im akademischen Überlebenskampf zu verschaffen). ${ }^{4}$ In diesem Kontext ist es wenig überraschend, dass sich über $70 \%$, darunter insbesondere die Jüngeren, der im Verein für Sozialpolitik organisierten deutschprachigen ÖkonomInnen explizit zur Neoklassik oder verwandten Ansätzen bekennen (Frey, Humbert und Schneider 2007: 361-364).

Diese Marginalisierung alternativer Theorien hat Konsequenzen nicht nur für die Ökonomie als Disziplin, sondern auch für die Praxis linker Politikprojekte. Progressive Reformpolitik ist in besonderem Maße angewiesen auf sozialwissenschaftliche Erkenntnisse: weil sie sich empirisch überprüfbare Ziele setzt, braucht sie seriöse sozialwissenschaftliche Forschung sowohl zur Gestaltung ihrer Maßnahmen als auch zu deren Evaluation, um gegebenenfalls einen eingeschlagenen Kurs zu korrigieren. Die Offenheit für sozialwissenschaftliche Erkenntnisse ist

2 Die Bezeichnung verschiedener theoretischer Strömungen innerhalb der Ökonomie orientiert sich an einer dem Kirchenlatein entlehnten Terminologie: Die institutionell und personell weitgehende dominierende Neoklassik gilt als „Orthodoxie“ (richtiger Glaube), die verschiedenen alternativen Strömungen, von evolutionär über keynesianisch und marxistisch bis hin zu ökologisch, gelten als ,heterodox“ (falsch- bzw. andersgläubig).

3 Neben der innerdisziplinären „Selbstbereinigung“ existiert auch eine Tendenz der Universitätsverwaltungen - bedingt durch den Wettbewerb um talentierte Studierende, Fördermittel, Subventionen und Personal - heterodoxe Organisationseinheiten zu Gunsten der in Rankings besser platzierten Mainstream-Ökonomie zu ersetzen. Dieser Anreiz existiert auch dann, wenn heterodoxe Forschung auf hohem Niveau durch neoklassische Forschung auf mittelmäßigem Niveau ersetzt werden kann (vgl. Glenn 2009 für ein aktuelles Fallbeispiel und Lee 2010 für eine allgemeine Darstellung).

4 Die Petition wurde von 83 Personen, vorwiegend ProfessorInnen der Volkswirtschafslehre, unterzeichnet und erschien in der Frankfurter Allgemeinen Zeitung vom 5. Mai 2009. Ein daraufhin veröffentlichter Gegenappell wurde von 188 ÖkonomInnen unterzeichnet und erschien unter dem Titel „Baut die deutsche VWL nach internationalen Standards um!“ am 8. Juni im Handelsblatt (siehe auch die scharfsinnigen Kommentare zu dieser Kontroverse in Rothschild 2010). 
dabei ebenso sehr eine Stärke reformistischer Ansätze, wie sie auch Einfallstor für Irrtümer und (selektive) Blindheit der herrschenden sozialwissenschaftlichen Paradigmen darstellt.

In ihrer derzeitigen, inhaltlichen, methodischen und institutionellen Verfasstheit kann ein großer Teil der Wirtschaftswissenschaft jedoch keinen Beitrag leisten, um progressive oder gar transformative Reformpolitik zu informieren. Es ist im Gegenteil vielmehr so, dass die Überwindung eben dieses wirtschaftswissenschaftlichen Mainstreams selbst eine der größten Herausforderungen für linke Reformpolitik darstellt. Das Problem ist dabei nicht die Existenz neoklassischer Ansätze, sondern ihre Vorherrschaft, ihr Anspruch die Wirtschaftswissenschaft schlechthin zu repräsentieren. Denn es ist kein Zufall, dass sich alternative ökonomische Paradigmen häufig auch um alternative Werturteile strukturieren, die etwa Nachhaltigkeit (ökologische Ökonomie), Gleichberechtigung der Geschlechter (feministische Ökonomie) oder gerechte Verteilung und hohe Beschäftigung (Post-Keynesianismus) als erstrebenswert betrachten. ${ }^{5}$

Ein Ausweg aus dieser Misere ist deshalb auch nicht die bloße Ersetzung des einen Paradigmas durch ein anderes. Angesichts steigender Komplexität und Dringlichkeit globaler Fragestellungen in Bereichen wie Klima, Verteilung und Menschenrechte kann Politik nicht nur auf ein Pferd zu setzen. Sie ist auf die befruchtende Auseinandersetzung verschiedenster sozialwissenschaftlicher Theorien und Methoden geradezu angewiesen. Dies gilt für heterodoxe Traditionen in der Ökonomie wie (post-)keynesianische, evolutionäre oder marxistische Ansätze ebenso wie für organisations- und wirtschaftssoziologische Perspektiven. Nicht zuletzt gilt dieser grundsätzliche Gedanke auch für die neoklassische Ökonomie, obgleich ihrer offensichtlich tendenziell wirtschaftsliberalen ideologischen Konnotationen. Das Problem ist hier weniger ein ideologisches, sondern ein institutionelles, nämlich dass die aktuelle paradigmatische Verfasstheit neoklassischen Denkens mit einem pluralistischen Diskurs nicht kompatibel ist.

Im Ergebnis würde eine derart pluralistische Sozial- und Wirtschaftswissenschaft ein Ende der einfachen Antworten bedeuten und die Vorwegnahme politischer Entscheidungen durch Expertenkommissionen erschweren. Sozialwissenschaft dabei als genuin politisches Unterfangen zu begreifen, ist sowohl aufSeiten der Wissenschaft als auch aufSeiten der Politik die Voraussetzung für einen derartigen Wandel.

5 Dies bedeutet freilich nicht, dass sich unterschiedliche ökonomische Paradigmen nur durch ihre differente ideologische Ausrichtung unterscheiden; es soll vielmehr heißen, dass (a) jedes ökonomische Paradigma auch (mehr oder weniger unterschiedliche) ideologische Implikation mit sich bringt und (b) die Ökonomie daher auch institutionell von politischen Motiven beeinflusst wird. 
Im Rahmen dieses Papiers versuchen wir uns an einer systematischen Analyse der Gründe für die Rigidität und den methodischen Monismus der Ökonomie sowie die Schwierigkeiten diesen zu überwinden, bevor wir daran anschließend zwei zumindest teilweise komplementäre Handlungsstrategien für mögliche Auswege skizzieren.

\section{Ursachen neoklassischer Dominanz in der Ökonomie}

Angesichts der z.B. in den Gutachten des überwiegend mit neoklassischen ÖkonomInnen besetzten Sachverständigenrats für Wirtschaft seit Jahrzehnten dokumentierten Kontinuität und Einseitigkeit wirtschaftswissenschaftlicher Politikempfehlungen stellt sich die Frage nach den Ursachen der paradigmatischen Dominanz neoklassischer Ökonomie. Nachdem diese wohl kaum mittels einer prinzipiellen inhaltlichen Überlegenheit der neoklassischen Theorie gerechtfertigt werden kann (Keen 2001), scheinen im Kontext der paradigmatischen Dominanz neoklassischen Denkens vor allem institutionelle Faktoren von Bedeutung zu sein.

Diese institutionellen Faktoren - so die hier artikulierte These - führen zu sich wechselseitig verstärkenden Feedback-Effekten, die zu einer Stabilisierung der Dominanz neoklassischer Theorie auf nicht zu unterschätzende Weise beitragen. ${ }^{6}$ Derartige positive Rückkoppelungseffekte zwischen den institutionellen Rahmenbedingungen an Universitäten und Forschungseinrichtungen und der dominanten Stellung neoklassischer Ansätze finden sich dabei zumindest im Bereich (1) der Karrieremöglichkeiten (Publikationen, Anstellungen), (2) der universitären Lehre und (3) der innerdisziplinären Diskurskultur. Erschwerend kommt hinzu, dass sich (4) der neoklassische Mainstream innerhalb der Ökonomie bewusst von alternativen Ansätzen - und damit auch von potentieller Kritik oder möglichen Innovationen aus diesem Bereich - abgrenzt. Diese Abgrenzung wird in Form von (Nicht-)Zitationen sichtbar und verstärkt so - vermittelt bzw. institutionalisiert durch Zitationsrankings als wichtigstem Evaluationsinstrument - wiederum die zuvor genannten Punkte (1) - (3).

Der erste Punkt ist weitgehend offensichtlich: Eine Karriere als ÖkonomIn ist ohne Minimalbekenntnis zur Neoklassik heute kaum mehr vorstellbar. Die grundsätzliche Bedingung für eine Einstellung an einer deutschsprachigen Universität im post-doktoralen Bereich besteht im Normalfall darin, zumindest in Randbereichen der Mainstream-Ökonomie anschlussfähig zu sein. Auch

6 Die Dominanz der neoklassischen Theorie wird hier vorausgesetzt. Vgl. zur historischen Genese dieser Dominanz Dobusch/Kapeller (2009a), Walpen (2004). 
auf eine primär „theoriefreie“, statistisch-ökonometrische Qualifikation kann mitunter erfolgreich verwiesen werden. Schwieriger wird es hingegen, wenn in den Forschungsschwerpunkten ein klares Bekenntnis zu einer heterodoxen Theorie oder ein Schwerpunkt auf der Geschichte des ökonomischen Denkens ausgewiesen wird. Dies bedeutet nicht, dass so qualifizierte ForscherInnen niemals eine Anstellung finden können, sondern bloß, dass deren Karriereoptionen wesentlich eingeschränkt sind - schließlich stellen ökonomische Institute, die dezidiert nach alternativ geschulten ForscherInnen suchen, die große Ausnahme dar. ${ }^{7}$ Hier kommt es also zu einem sehr simplen selbstverstärkenden Effekt, da jene Personen, die innerhalb der Ökonomie beruflich vorankommen wollen, gut beraten sind, sich bereits zur Vorbereitung eingehend mit der neoklassischen Theorie zu befassen und sich nicht von Alternativen zur oder Kritiken an der Neoklassik verwirren oder ablenken zu lassen - ansonsten wird der Karriereweg unter Umständen steinig. ${ }^{8}$

Ähnliches gilt auch für die Publikationsmöglichkeiten: In den prestigeträchtigsten Journalen der ökonomischen Disziplin ist eine Anknüpfung an neoklassische Denklinien ohnehin nahezu unumgänglich und auch in den Journalen der „zweiten und dritten Reihe“ gehört dies, mit wenigen Einschränkungen, zum Standard, an dem ein eingereichter Artikel gemessen wird. Dies ist auch ein wesentlicher Grund für die Herausbildung dezidiert heterodoxer Journale (zu den bekanntesten zählen das Cambridge Journal of Economics, das Journal of Economic Issues, das Journal of Post-Keynesian Economics und Ecological Economics; vgl. zu diesem Themenkomplex auch Dobusch/Kapeller 2009c). In diesem Sinne ist die Ursache für die Gründung eines heterodoxen Journals oft im Ausschluss aus der Mainstream-Literatur zu suchen (vgl. King 2002: 134-136; Reardon 2008).

„Die Konfrontation Heterodoxie kontra Mainstream bezieht ihre Existenz und ihre Berechtigung vielmehr aus dem gegenwärtigen Zustand des Wissenschaftsregimes im ökonomischen Bereich, das durch eine unübersehbare Bevorzugung und Förderung eines Mainstreams neoklassischer Prägung an Universitäten, Forschungsinstituten und staatlichen und internationalen Wirtschaftsorganisationen charakterisiert ist. Konzentration auf diese Richtung wird im Studium gefördert und spielt eine entscheidende Rolle für

7 Diese Universitäten bzw. Institute decken sich zum Teil mit jenen, die laut dem „Informational Heterodox Directory“ auch alternative Ausbildungsstränge anbieten (vgl. http://heterodoxnews.com/directory). Im deutschsprachigen Raum sind hier noch die Universität Bremen und die HTW Berlin gelistet.

8 Auch für theoretische Argumente gilt ähnliches. Wie Sent (2004) zeigt, wurde die Aufnahme psychologischer Argumente in die neoklassische Theorie (unter dem Stichwort behavioral economics) vor allem dadurch ermöglicht und erleichtert, dass sie als Ergänzung $\mathrm{zu}$ - und nicht als Kritik an - den gängigen theoretischen Vorstellungen präsentiert wurden. 
Beruf und Karriere. Von dieser Schieflage sind alle nicht-neoklassischen und kritischen Richtungen betroffen, was die Herausbildung der Heterodoxie-Terminologie und der Heterodoxie-Bewegung erklärt." (Rothschild 2008: 25)

In der ökonomischen Lehre wiederum sind, zweitens, die paradigmatischen Vorgaben nochmals enger und unflexibler als im Bereich der Forschung (Wilson/ Dixon 2009). Die zentralen Lehrinhalte werden durch eine global homogenisierte Lehrbuchkultur vorgegeben. Den Ausgangspunkt dieser Homogenisierung bildet Paul Samuelsons epochales Lehrbuch (Samuelson 1973, erste Auflage 1948) als zentraler Blueprint: Erfolgreiche Lehrbücher folgen in Struktur, Aufbau und Inhalt im Wesentlichen dem Samuelson'schen Vorbild, ganz unabhängig von Verlag oder AutorIn (Stiglitz 1988, Lee/Keen 2004, Ötsch/Kapeller 2010)ํ: „Most new textbooks are, generally speaking, clones of existing ones. „(Hill/ Myatt 2009: 58)

Hier bleibt nur noch zu ergänzen, dass diese Lehrbücher im Wesentlichen oder zur Gänze die neoklassische Theorie abdecken, während alternative Theorieansätze unberücksichtigt bleiben. Derartige Ansätze müssen also von den Studierenden selbst erarbeitet werden und sind so, wenn überhaupt, nur am Rande des Regelstudiums präsent. Hier ergibt sich ein ähnlicher Effekt wie zuvor: Zum einen werden auch jene Personen, die Ökonomie nur als Nebenfach absolvieren, mit einem gewissen neoklassischen Grundwissen ausgestattet, bevor sie in ihre eigentlichen Berufe als Angestellte, BeamtInnen, LehrerInnen oder JournalistInnen einsteigen. Die neoklassische Theorie wird so zum Teil einer sozialwissenschaftlichen Allgemeinbildung. Zum anderen sind aufgrund des hier skizzierten Ausbildungsdesigns auch ein Großteil der spezialisierten AbsolventInnen in Ökonomie primär oder exklusiv in der neoklassischen Theorie geschult - Nachfrage wie Angebot an professionellen ÖkonomInnen ist damit stark neoklassisch geprägt und führt zu einem sich selbst verstärkenden Rückkoppelungseffekt: Mehr Neoklassik in der Ausbildung führt zu mehr neoklassisch geschulten Personen auf dem Arbeitsmarkt und - bei deren Anstellung - wiederum zu mehr Neoklassik in der Ausbildung etc.

Vor dem Hintergrund dieser Konstellation, die einen prägenden Einfluss des Paradigmas auf den jeweiligen Arbeitsmarkt hat, ist es wenig überraschend, dass

9 Auch hier machen sich wieder selbstverstärkende Mechanismen bemerkbar: Vorschläge für Lehrbücher, die nicht dem etablierten Standard entsprechen, erhalten in den der Publikation vorgelagerten Review-Verfahren systematisch schlechtere Bewertungen, da die Orientierung am Samuelson'schen blueprint auch für die jeweiligen Gutachterinnen ein zentrales Kriterium ist (siehe Ötsch/ Kapeller 2010: 18 für ein konkretes Beispiel). Alternative Lehrbücher, die diesen impliziten Vorgaben nicht folgen, finden sich ebenso in Ötsch/Kapeller (2010: 23). 
die Zustimmung zur Neoklassik gerade bei jüngeren ÖkonomInnen überproportional ansteigt (Frey et al. 2007). Das Paradigma reproduziert sich hier im Zusammenspiel von einseitiger Ausbildung, stromlinienförmigen Karriereoptionen und dem Ehrgeiz junger WissenschaftlerInnen.

Die Enge der neoklassischen Diskurskultur geht schließlich, drittens, mit einer Reihe weiterer, eher subtiler Aspekte einher, die ebenso geeignet sind die dominante paradigmatische Stellung der neoklassischen Theorie zu reproduzieren. Zwei Elemente sind hier von besonderer Bedeutung, nämlich einerseits der Bezug der Neoklassik auf gewisse traditionell etablierte Denkfiguren, wie Knappheit, Gleichgewicht, Optimierung, Rationalität oder Individualismus und andererseits die implizite Voraussetzung eines methodischen Stils, der vor allem durch die Verwendung mathematischer Modelle und ihre statistisch-ökonometrische Schätzung definiert ist. Beide Aspekte sind geeignet, konkurrierende Theorien oder widersprechende empirische Evidenz aus der Sphäre des ökonomischen Diskurses zu verweisen: Wer die für die neoklassische Ökonomie typischen Denkfiguren und Routinen ignoriert, der oder die sei nämlich gar kein Ökonom, sondern eher Betriebswirt, Soziologe oder Scharlatan (drei Begriffe, die aus Sicht vieler ÖkonomInnen weitgehend zusammenfallen).

Vielen bekannten kritischen ÖkonomInnen, darunter etwa John K. Galbraith oder Joan Robinson, wurde auf dieser Basis ihre Berechtigung „ökonomisch $\mathrm{zu}$ argumentieren“ abgesprochen. Diese Vorgehensweise hat einen doppelten Vorteil: Zum einen muss man sich mit innerdisziplinärer Kritik dieser Art nicht weiter befassen - sie kommt ja, in neoklassischer Wahrnehmung, ohnedies von außerhalb der Disziplin - und zum anderen wird alternativen ökonomischen Sichtweisen kein prestigeträchtiges Terrain überlassen: Es mag ja sein, dass solche Persönlichkeiten irgendeine Art von politikrelevanter Expertise anzubieten hätten, es kann sich dabei aber eben nicht um „ökonomische Expertise“ handeln.

Darüber hinaus bietet vor allem die mit der „ökonomischen Methode“ assoziierte typische Abfolge von Elementen eines wissenschaftlichen Artikels Aufstellen von Annahmen, Untersuchung eines daraus resultierenden mathematischen Modells und eventuell ein nachgelagerter, auf Verifikation abzielender empirischer „Test“ - ein ideales Kochrezept zur Erstellung publikationsfähiger ökonomischer Artikel und zugleich ein Demarkationskriterium zur Unterscheidung von Ökonomie und Nicht-Ökonomie. In diesem Sinne markiert die ökonomische Methode die Grenzen des neoklassischen Paradigmas - wer diese überschreitet, verliert dabei seine Berechtigung ,als ÖkonomIn“ zu sprechen. Dass ein solcher Ansatz - vor allem durch die Verbindlichkeit der mathematischen Formulierung - dazu tendiert, gewisse theoretische Ansätze zu ignorieren (etwa jene, die nicht, noch nicht oder nicht sinnvoll in eine formale Sprache übersetzt werden können) und andere zu verändern oder gar zu verfälschen (Backhouse 
1998, Lagueux 2004), wird von der neoklassischen Community dabei bereitwillig in Kauf genommen.

Vor dem Hintergrund des bisher Gesagten ist es nicht weiter überraschend, dass neoklassische ÖkonomInnen, viertens, auf eine Kommunikation mit konkurrierenden Paradigmen weitgehend verzichten - ein Umstand, der die dominante Stellung der Neoklassik weiter stärkt, da alternative oder kritische Sichtweisen so automatisch noch weniger Raum erhalten. Eine Analyse der Zitationspraxen führender neoklassischer wie auch heterodoxer Journale zeigt klar, dass sich letztere zwar intensiv mit der Mainstreamtheorie beschäftigen, dieses Verhalten allerdings keinen reziproken Gedankenaustausch auslöst. Ganz im Gegenteil: Während die Heterodoxie den neoklassischen Mainstream brav rezipiert und sich mit diesem auseinandersetzt, werden heterodoxe Ideen in MainstreamJournalen nur sehr selten rezipiert - und wenn dann oft ohne entsprechende Literaturverweise zu setzen.

Die folgende Tabelle basiert auf einer Untersuchung der Zitationspraxen von 26 führenden Ökonomie-Journalen (davon 13 Mainstream-Journale und 13 heterodoxe Journale) im Zeitraum von 1989 bis 2008 und verdeutlicht diesen Zusammenhang. Sie zeigt, dass die heterodoxen Journale weitaus mehr Zitate aus dem Mainstream importieren, als sie in selbigen exportieren.

Diese folgende Tabelle verdeutlicht, dass der oben beschriebene komparative Vorteil des neoklassischen Paradigmas noch einmal verstärkt wird, da heterodoxe Journale Mainstream-Journale um den Faktor 9,34 häufiger zitieren als umgekehrt. Dies bedeutet, dass die heterodoxe Ökonomie den aktuellen Mainstream rankingtechnisch stärkt - und zwar dank der inhaltsblinden Logik des Zitatezählens, auch dann, wenn die entsprechenden Zitate eingesetzt werden, um gewisse Ansichten zu kritisieren. ${ }^{10}$

Besonders dramatisch ist dieser Zusammenhang, da die - auf eben diesen Zitationen basierenden - Rankings von Journalen, AutorInnen und Departments

10 Auffallend ist auch, dass die absolute Summe der vom Mainstream importieren Zitate, mit 753 in 20 Jahren, eher niedrig ist. Betrachtet man diesen Wert genauer, so stellt man fest, dass mehr als vier Fünftel dieser Zitate auf die jeweiligen „Ausreißer“ zurückzuführen sind. So exportiert das Journal of Economic Behavior and Organization (JEBO), als einziges in beiden Feldern beliebtes Journal, 340 Zitate, während die beiden Ausreißer auf der Seite der Mainstream-Ökonomie, das Journal of Economic Geography und Economic Geography, die heterodoxen Journale (exlusive JEBO) insgesamt 273 mal zitieren. Es verbleiben also nur 140 Zitate in 20 Jahren (also sieben Zitate/Jahr), die von den verbleibenden zwölf heterodoxen Journalen in die restlichen elf Mainstream-Journale exportiert werden. Dies zeigt, dass de facto kein durch Zitationsflüsse nachweisbarer Ideentransfer von den relativ anerkanntesten Journal der heterodoxen Ökonomie zu etablierten Mainstream-Journalen existiert. 
Tabelle 1: Zitations-„Handelsbilanzen“zwischen Heterodoxie und Orthodoxie

\begin{tabular}{|c|c|c|c|c|}
\hline Top 13 „,heterodox“ & $\begin{array}{c}\text { Summe der in } \\
\text { den Mainstream } \\
\text { exportierten Zitate }\end{array}$ & $\begin{array}{c}\text { Summe der aus } \\
\text { dem Mainstream } \\
\text { importierten Zitate }\end{array}$ & Differenz & $\begin{array}{l}\text { Quotient: } \\
\text { Importel } \\
\text { Exporte }\end{array}$ \\
\hline Economy and Society & 46 & 69 & -23 & 1.5 \\
\hline Ecological Economics & 18 & 1022 & -1004 & 56.78 \\
\hline $\begin{array}{l}\text { Work, Employment } \\
\text { and Society }\end{array}$ & 17 & 47 & -30 & 2.76 \\
\hline $\begin{array}{l}\text { Review of international } \\
\text { Political Economy }\end{array}$ & 55 & 111 & -56 & 2.02 \\
\hline $\begin{array}{l}\text { Journal of Econo- } \\
\text { mic Behaviour and } \\
\text { Organization }\end{array}$ & 340 & 2605 & -2265 & 7.66 \\
\hline New Political Economy & 5 & 50 & -45 & 10 \\
\hline $\begin{array}{l}\text { Cambridge Journal of } \\
\text { Economics }\end{array}$ & 98 & 617 & -519 & 6.3 \\
\hline $\begin{array}{l}\text { Journal of Develop- } \\
\text { ment Studies }\end{array}$ & 72 & 672 & -600 & 9.33 \\
\hline $\begin{array}{l}\text { Journal of Evolutionary } \\
\text { Economics }\end{array}$ & 36 & 517 & -481 & 14.36 \\
\hline Feminist Economics & 7 & 198 & -191 & 28.29 \\
\hline $\begin{array}{l}\text { Journal of Post- } \\
\text { Keynesian Economics }\end{array}$ & 10 & 407 & -397 & 40.7 \\
\hline $\begin{array}{l}\text { Journal of Economic } \\
\text { Issues }\end{array}$ & 22 & 568 & -546 & 25.82 \\
\hline $\begin{array}{l}\text { Economics \& } \\
\text { Philosophy }\end{array}$ & 27 & 153 & -126 & 5.67 \\
\hline Summe & 753 & 7036 & -6283 & 9.34 \\
\hline
\end{tabular}

Quelle: Dobusch und Kapeller 2009c. Die dieser Analyse zu Grunde liegenden Daten stammen aus dem Thomson Scientifics „Web of Science,“ die auch die Datenbasis zur Kalkulation von Impact-Faktoren und den dazugehörigen Journal-Rankings darstellt und erfassen alle Zitationsbeziehungen zwischen diesen 26 Journalen im Zeitraum von 1989 bis 2008.

ihrerseits wieder einen immensen Einfluss auf den Wissenschaftsbetrieb nehmen. Schließlich werden diese Rankings sehr häufig als Grundlage für eine ganze Reihe von institutionell bedeutenden Entscheidungen herangezogen: etwa im Bereich der Personalauswahl, der Bewilligung von Förderungen oder der Zuteilung von Lehraufträgen. ${ }^{11}$ Eine solche Praxis ist zwar methodologisch kaum haltbar, da

11 Hier gilt der Zusammenhang, dass je höher die Platzierung in den Rankings umso kleiner die Anzahl der zu leitenden Lehrveranstaltungen. Gut gerankte Forschungsleistungen führen also zu einer Reduktion des Lehraufwands der Betroffenen. In manchen Kontexten (etwa in Australien, siehe King/Kriesler 2008) ist diese Praxis direkt in die universitären 
sich zeigen lässt, dass derartige Rankings nicht die Qualität, sondern vielmehr den Einfluss von Artikeln erfassen (siehe Kapeller 2010), aber nichtsdestotrotz in der universitären Praxis und hier wiederum insbesondere in der Ökonomie weitverbreitet. Es kommt also zu einem rekursiven Verstärkungseffekt, da innerdisziplinärer Einfluss zuerst via Rankings gemessen wird und dann - auf Basis eben derselben Rankings - von Neuem verteilt wird. Hier gilt dann letztlich das Matthäus-Prinzip in seiner ursprünglichen Form: „Wer (Einfluss) hat, dem wird (noch mehr Einfluss) gegeben" (vgl. schon Merton 1968).

Darüber hinaus zeigt eine solche Analyse auch, dass die heterodoxe Ökonomie im Vergleich zum Mainstream strategisch sehr ungeschickt agiert: Neben der überproportionalen Berücksichtigung des paradigmatischen Konkurrenten der Mainstreamökonomie - ist auffallend, dass die verschiedenen heterodoxen Strömungen untereinander kaum kommunizieren. Dies verdeutlichen die nachstehenden beiden Tabellen, die auf derselben Datengrundlage wie Tabelle 1 basieren.

Tabelle 2: Orthodoxe und Heterodoxe Zitationsnetzwerke

\begin{tabular}{lcc}
\hline & $\begin{array}{c}\text { Durchschnittlicher Anteil der } \\
\text { Zitate aus den Top 13 heterodoxen } \\
\text { Journalen }\end{array}$ & $\begin{array}{c}\text { Durchschnittlicher Anteil der } \\
\text { Zitate aus den Top 13 orthodoxen } \\
\text { Journalen }\end{array}$ \\
\hline $\begin{array}{l}\text { in den Top 13 } \\
\text { „heterodox“ }\end{array}$ & $52.42 \%$ (netzwerkintern) & $47.58 \%$ (netzwerkübergreifend) \\
\hline $\begin{array}{l}\text { in den Top 13 } \\
\text { „orthodox" }\end{array}$ & $2.85 \%$ (netzwerkübergreifend) & $97.15 \%$ (netzwerkintern) \\
\hline
\end{tabular}

Quelle: Dobusch/Kapeller 2009c

Tabelle 3: Die Rolle von Journal-Selbst-Zitaten ${ }^{12}$ in orthodoxen und heterodoxen Zitationsnetzwerken.

\begin{tabular}{lcc}
\hline & $\begin{array}{c}\text { Anteil der netzwerkinternen } \\
\text { Zitate (heterodox und orthodox) } \\
\text { exklusive Journal-Selbst-Zitate }\end{array}$ & $\begin{array}{c}\text { Anteil der Journal-Selbst- } \\
\text { Zitate an den netzwerk- } \\
\text { internen Zitaten }\end{array}$ \\
\hline in den Top 13 „heterodox“ & $13.46 \%$ (netzwerkintern) & $71.71 \%$ \\
\hline in den Top 13 „orthodox“ & $68.79 \%$ (netzwerkintern) & $29.19 \%$ \\
\hline
\end{tabular}

Quelle: Dobusch/Kapeller 2009c

Institutionen integriert, in anderen (z.B. in Deutschland) äußert sie sich indirekt, etwa an den unterschiedlichen Lehrvolumina für Universitäts- und Fachhochschulprofessuren. Letztere sind dabei auch ÖkonomInnen zugänglich, die in den Rankings schlechter platziert sind, und im Normalfall mit einem weitaus höheren Lehraufwand verbunden.

12 Unter Journal-Selbst-Zitaten versteht man Zitate, die auf einen anderen Artikel im selben Journal verweisen. 
Tabelle 2 zeigt, dass Journale der Mainstream-Ökonomie vorwiegend andere Mainstream-Journale zitieren, während heterodoxe Journale sowohl andere heterodoxe Inhalte als auch Argumente der Mainstreamökonomie in einem relativ ausgewogenen Verhältnis rezipieren. Der neoklassische Diskurs erscheint hier als geschlossen für die paradigmatische Konkurrenz, während der heterodoxe Diskurs auch für neoklassische Argumente weitgehend offen ist - wenn auch vor allem in Form von Kritik an ebendiesen Argumenten.

In Tabelle 3 wird hingegen versucht, die netzwerkinternen Kommunikationsroutinen zu reflektieren. Es zeigt sich, dass - in starkem Gegensatz zur Mainstream-Ökonomie - der netzwerkinterne heterodoxe Diskurs zu einem großen Teil (etwa 70\%) auf Journal-Selbst-Zitationen beruht. Dies impliziert, dass unterschiedliche heterodoxe Traditionen - neben ihrer Auseinandersetzung mit dem Mainstream - vor allem mit sich selbst kommunizieren, ihre potentiellen „Alliierten,“ also die anderen heterodoxen Traditionen, aber weitgehend ignorieren. Diese „diskursive Selbstisolation“ führt dazu, dass dem ohnehin schon übermächtigen neoklassischen Block keine institutionell oder diskursive geschlossene Heterodoxie gegenübersteht, sondern vielmehr ein Flickenteppich alternativer Ideen, Konzepte und Ansätze, die ihrerseits wiederum nur eine lose Bindung aufweisen. Diese fehlende institutionelle Kohärenz der Heterodoxie macht es natürlich umso leichter, die in einem heterodoxen Kontext vorgebrachten Argumente, Theorien und Politikvorschläge zu ignorieren. Die heterodoxe Ökonomie erscheint aus dieser Perspektive als viel zu partikularistisch, um in einem Vergleich mit dem wesentlich geschlosseneren neoklassischen Diskursnetzwerk auch nur ansatzweise bestehen zu können.

Zusammengefasst lässt sich an Hand der vier in diesem Abschnitt ausgeführten Punkte - Karrierevorteile durch Nähe zum Mainstream, neoklassisch dominierte Lehre, innerdisziplinär-diskursive Herrschaftsstrategien sowie deren Institutionalisierung in Form von Zitationsrankings - eine sich selbst verstärkende Vormachtstellung des neoklassischen Paradigmas in der Ökonomie feststellen, die durch unreflektiertes bzw. unkoordiniertes Handeln heterodoxer ÖkonomInnen noch verstärkt wird. Für progressive Reformpolitik sind mit dieser Dominanz aber, wie bereits im vorhergehenden Abschnitt illustriert, große Probleme verbunden, da ökonomische ExpertInnenmacht so in der Regel eine ideologisch marktliberale Schlagseite aufweist. Angesichts der wachsenden Bedeutung von expertokratischen (Vor-)Entscheidungsprozeduren - insbesondere auch im Bereich transnationaler Politikprozesse - ist deshalb eine Änderung der paradigmatischen Verfasstheit der Ökonomie selbst eine der Hauptaufgaben progressiver Reformpolitik. Welche Strategien hierfür verfolgt werden könnten, ist Thema des folgenden, letzten Abschnitts. 


\section{Strategien zur Pluralisierung ökonomischer Wissenschaft}

Prinzipiell lassen sich zwei Ansatzpunkte festmachen, um die Folgen der derzeitigen Verfasstheit der ökonomischen Disziplin für progressive Reformpolitik zu reduzieren: (a) innerdisziplinäre und (b) außerdisziplinäre. Die Vorschläge sind dabei von der Überzeugung getragen, dass eine Pluralisierung des ökonomischen Diskurses sowohl zu einer besseren ökonomischen Forschung führen als auch bzw. schon alleine dadurch die Voraussetzungen für erfolgreiche progressive Politikprojekte verbessern würde. Gleichzeitig sind diese Vorschläge angesichts des zuvor beschriebenen Ausmaßes institutioneller Rigidität der Ökonomie aber in erster Linie Überlebensstrategien für die marginalisierte Minderheit heterodoxer ÖkonomInnen und auch in ihrer Gesamtheit kaum geeignet, ohne die Veränderung gesellschaftlicher und politischer Rahmenbedingungen, einen grundlegenden Wandel herbeizuführen.

\section{(a) Innerdiszplinäre Strategien}

Zu den wichtigsten innerdisziplinären Strategien gehört die Stärkung heterodoxer Ansätze durch die Einrichtung bzw. Förderung von Forschungsinstituten und (Stiftungs-)Lehrstühlen mit heterodoxer Ausrichtung - und zwar unbesehen einer unmittelbar progressiv-politischen Ausrichtung. Als Beispiele können hierfür in Deutschland das aus Mitteln der Hans-Böckler-Stiftung finanzierte Institut für Makroökonomie und Konjunkturforschung (IMK) oder, im Rahmen der Max-Planck-Gesellschaft, das Max-Planck-Institut für evolutionäre Ökonomie in Jena gelten. Es bestehen durchaus auch Möglichkeiten auf regionaler Ebene tätig zu werden, wie in Österreich das jüngst aus Mitteln der Stadt Linz finanzierte und an der Universität Linz angesiedelte Institut für die Gesamtanalyse der Wirtschaft (ICAE) beweist (vgl. http://www.icae.at/).

Letzteres ist auch noch aus einem anderen Grund ein interessantes Beispiel, ist es im Unterschied zu IMK und dem MPI in Jena prinzipiell theorieoffen angelegt und leistet somit einen Beitrag zur dringend notwendigen Stärkung der inhaltlichen Auseinandersetzung zwischen verschiedenen heterodoxen Schulen. Wie im vorhergehenden Abschnitt ausgeführt, wäre eine stärkere Vernetzung und - auch: kritische - Auseinandersetzung zwischen verschiedenen heterodoxen Ansätzen nicht nur aus forschungslogischen Überlegungen sinnvoll, sondern auch aus pragmatischen Zitationsüberlegungen heraus. In diesem Sinne wäre eine Bündelung heterodoxer Kompetenzen im Rahmen gemeinsamer (Zusatz-) Ausbildungsangebote wie Master-Lehrgänge oder Summer-Schools sinnvoll. Derzeit sind solche Angebote, sofern sie überhaupt existieren, meist streng nach Schulen getrennt. Schließlich könnte auch die Etablierung eines Journals of 
Heterodox Economics einen Beitrag zur Förderung einer vergleichend-kritischen Auseinandersetzung innerhalb der heterodoxen Ökonomie leisten.

Zusammengenommen zielen innerdisziplinäre Pluralisierungsstrategien einerseits auf eine Stärkung heterodoxer Ansätze und andererseits auf eine Intensivierung der inhaltlichen Auseinandersetzung zwischen ebendiesen Ansätzen. Wie wichtig diese Strategie der kleinen Schritte zu sein scheint, illustriert die Reaktion des ökonomisch-akademischen Establishements auf die jüngste Finanzkrise. Obgleich die neoklassische Forschungsgemeinschaft durchaus als mitverantwortlich für Entstehung und Ausmaß der Krise angesehen wird - und das zum Teil auch innerhalb der Mainstream-Ökonomie (siehe etwa Colander et al. 2009) - bleiben größere institutionelle oder forschungsstrategische Änderungen innerhalb dieses dominanten Paradigmas weitgehend aus. Der Hauptgrund hierfür ist freilich die Selbstreferentialität des akademischen Diskurses, dessen Qualitätskritierien sich durch die Finanzkrise keineswegs verschoben haben. Der Statusverlust neoklassischer Ökonomie, so dieser überhaupt feststellbar ist, bezieht sich also primär auf den öffentlichen, aber nicht auf den innerakademischen Diskurs. Und in diesem geht es, zumindest für jene kleine Teilmenge kritischer ÖkonomInnen, weniger um eine Herausforderung der Mainstream-Ökonomie, sondern vielmehr um das eigene Überleben in einer Welt des 'publish or perish.' Solange sich diese, in gewissem Sinne hochgradig prekäre Situation heterodoxer ÖkonomInnen nicht verbessert, bleibt ein grundsätzlicher paradigmatischer Wandel im Bereich der Ökonomie im Wesentlichen eine Illusion.

Um dies zu erreichen, ist daher auch eine stärkere Selbstorganisation jener Kräfte von Nöten, die in und rund um die Ökonomie tätig sind und dabei den kritischen Blick auf den neoklassischen Mainstream nicht verloren haben. Studentische Bewegungen, etwa nach dem Vorbild der französischen Bewegung für eine 'postautistische' Ökonomie, können zwar aller Wahrscheinlichkeit nach den Charakter der Disziplin nicht grundlegend ändern, sorgen aber für ein verstärktes kritisches Bewusstsein. Angesichts des tendenziell dogmatischen Charakters volkswirtschaftlicher Ausbildung (siehe etwa Albert 1998: 153), kann dieses kritische Bewusstsein als Grundlage einer Bereitschaft zur „,intellektuellen Selbstverteidigung “ gegenüber neoklassischen Prämissen und ihren Folgen verstanden werden. Derartige selbstorganisierte Projekte, wie etwa studentische Lesekreise, Initiativen zur Verbreiterung der Curricula oder das Verfassen kritischer Abschlussarbeiten sowie deren Publikation, bergen also ein nicht zu unterschätzendes kritisches Potential, das hilfreich erscheint, um letztlich zu einer pluralistischeren Konzeption ökonomischen Denkens zu gelangen. Eine der wenigen Schwachstellen des neoklassischen Bollwerks ist schließlich die, durch fehlende praktische Brauchbarkeit und zugleich hohe (formale) Schwierigkeit eines Ökonomie-Studiums bedingte, kleine Anzahl der Studierenden. Kann hier eine kritische Masse an entsprechend neoklassik- 
skeptischen Studierenden erreicht werden, wäre dies tatsächlich ein möglicher Vorbote paradigmatischen Wandels oder zumindest einer leicht gelockerten und weniger monolithischen Kultur an deutschsprachigen VWL-Instituten.

\section{(b) Außerdisziplinäre Strategien}

Ökonomische Wissenschaft und Expertise im Sinne eines empirisch fundierten Verständnisses wirtschaftlicher Zusammenhänge findet sich aber mehr denn je auch außerhalb der ökonomischen Disziplin. Mit Blick auf die empirische Fundierung sowie die diskursiv-hegemoniale Stärkung progressiver Reformpolitik bieten sich deshalb auch außerdisziplinäre Strategien an.

$\mathrm{Zu}$ diesen zählt jedenfalls die Förderung wirtschaftlicher Forschung in verwandten Disziplinen, insbesondere der Wirtschaftssoziologie bzw. der politischen Ökonomie. Eine VorreiterInnenrolle in dieser Hinsicht hat das Max-Planck-Institut für Gesellschaftsforschung in Köln inne. Um auch die außerwissenschaftliche Reputation dieser wissenschaftlich sehr anerkannten Forschungsströmungen zu stärken, würde es sich beispielsweise anbieten, für die Aufnahme von mindestens einem/r Nicht-Ökonomen/in in den bereits erwähnten Sachverständigenrat für Wirtschaft zu kämpfen.

Aber nicht nur außerdisziplinär, sondern auch außerhalb des Wissenschaftsbetriebs finden sich Handlungsfelder, in denen sich die negativen Folgen der neoklassischen Dominanz in der Ökonomie zumindest mildern lassen. Hierzu könnten einerseits die Förderung eines kritisch-heterodoxen Wirtschaftsjournalismus, aber auch junger heterodoxer ÖkonomInnen oder vielversprechender kritischer Abschlussarbeiten, über Stipendien und Preise sowie andererseits Aufklärungsarbeit über den problematischen Zustand der Ökonomie im Kontext von Parteien und parteinahen Einrichtungen, allen voran den Stiftungen, dienen. Denn insbesondere in den Doktorandenprogrammen der Stiftungen ließe sich die Förderung theoretischer und methodischer Vielfalt als Kriterium für die Förderung ökonomischer Abschlussarbeiten festschreiben sowie gezielt heterodoxe Ökonomen als Betreuungsdozenten anfragen.

Klar ist, dass viele dieser Gegenstrategien der Mitwirkung eben jener Minderheit an den Universitäten marginalisierter VertreterInnen von heterodoxen Ökonomischen Strömungen bedürfen. Umso wichtiger ist es, dass AkteurInnen außerhalb des Wissenschaftsbetriebs die Bedeutung (der Förderung) dieser heterodoxen ökonomischen Forschung erkennen und diese wo möglich unterstützen. Wahrscheinlich ist jedoch auch, dass selbst ein konsequentes Verfolgen dieser Strategien (alleine) noch nicht zu einem grundlegenden Wandel in der Ausrichtung der ökonomischen Disziplin führen würde, sondern auf komplementär-progressive Veränderungen der gesellschaftlichen Rahmenbedingungen angewiesen ist. 


\section{Literatur}

Albert, Hans (1998[1967]): Marktsoziologie und Entscheidungslogik. 2. Auflage, Tübingen: Mohr. Backhouse, Roger E. (1998): If Mathematics is informal, then perhaps we should accept that Economics must be informal too. Economic Journal, Vol. 108: 1848-1858.

Barro, Robert J. und Sala-i-Martin, Xavier (2004): Economic Growth. 2. Auflage. MIT Press.

Colander, David; Föllmer, Hans; Haas, Armin; Goldberg, Michael; Juselius, Katarina; Kirman, Alan; Lux, Thomas; Sloth, Brigitte. 2009. The Financial Crisis and the Systemic Failure of Academic Economics. University of Copenhagen Department of Economics Discussion Paper No. 09-03, URL: http://ssrn.com/abstract=1355882 (dl. 10.9.2009).

Crouch, Colin (2008): Postdemokratie. Frankfurt/M.: Suhrkamp.

Dobusch, Leonhard und Kapeller, Jakob (2009a): Why is Economics not an Evolutionary Science? New Answers to Veblen's Old Question. Journal of Economic Issues, 43(4): 867-898.

Dobusch, Leonhard und Kapeller, Jakob (2009b): Diskutieren und Zitieren: Zur paradigmatischen Konstellation aktueller ökonomischer Theorie. Intervention - Journal of Economics, 6(2): 145-152.

Dobusch, Leonhard und Kapeller, Jakob (2009c): A Guide to Paradigmatical Self-Marginalization: Lessons for Post-Keynesian Economists. Working paper. URL: http://www.dobusch.net/pub/ uni/Dobusch-Kapeller(2009)A_Guide_to_Paradigmatic_Self-marginalization-WP.pdf (dl. 28-12-10).

Frey, Bruno S., Humbert, Silke und Schneider, Friedrich (2007): Was denken deutsche Ökonomen? Eine empirische Auswertung einer Internetbefragung unter den Mitgliedern des Vereins für Socialpolitik im Sommer 2006. Perspektiven der Wirtschaftspolitik, 8(4): 359-377.

Glenn, David (2009): Notre Dame plan to dissolve its heterodox side of its split economics department. Chronicle of Higher Education vom 16. September 2009 URL: http://chronicle.com/ article/Notre-Dame-to-Dissolve/48460/(dl. 10-05-10)

Hill, Roderick und Myatt, Anthony (2007): Overemphasis on Perfectly Competitive Markets in Microeconomics Principles Textbooks. Journal of Economic Education, Vol. 38(1):58-77.

Kapeller,Jakob (2010): Citation metrics: serious drawbacks, perverse incentives and strategic options for heterodox economists. American Journal of Economics and Sociology, Vol. 69(5):1376-1408.

Kapeller, Jakob und Huber, Jakob (2009): Politische Paradigmata und neoliberale Einflüsse am Beispiel von vier sozialdemokratischen Parteien in Europa. Österreichische Zeitschrift für Politikwissenschaft, Vol. 38(2): 163-193.

Keen, Steve (2001): Debunking Economics. The naked emperor of the social sciences. London: Zed Books.

King, John E. (2002): A History of Post-Keynesian Economics since 1936. Cheltenham (UK): Edward Elgar.

King, John E., und Kriesler, Peter (2008): News from down under. On the Horizon, Vol. 16(4): 289-292.

Lagueux, Maurice (2004): The forgotten role of the rationality principle in economics.

Journal of Economic Methodology, Vol. 11(1): 31-51.

Lee, Frederic S. (2010): Pluralism in heterodox economics. In: Garnett, Robert, Olsen, Erik K. und Starr, Martha (Hrsg.): Economic Pluralism. London: Routledge: 19-35.

Lee, Frederic S, und Keen, Steve (2004): The Incoherent Emperor: A Heterodox Critique of Neoclassical Microeconomic Theory. Review of Social Economy, Vol. 62(2):169-199.

Lübbe, Weyma (2010): „Aus ökonomischer Sicht...“ - Was ist der normative Anspruch gesundheitsökonomischer Evaluationen? Rationality, Markets and Morals, Vol. 0(1): 451-463.

Merton, Robert K. (1968): The Matthew Effect in Science. In: Science, 159: 56-63

Ötsch, Walter O. (2009): Mythos Markt. Marburg: Metropolis.

Ötsch, Walter O. und Kapeller, Jakob (2010): Perpetuing the Failure: Economic Education and the Current Crisis. Journal of Social Science Education, Vol. 9(2): 16-25. 
Reardon, Jack (2008): Barriers to entry: heterodox publishing in mainstream journals. On the Horizon, Vol. 16(4): 185-197.

Rothschild, Kurt W. (2008): Apropos Keynesianer. In: Hagemann, Harald, Horn, Gustav und Krupp, Hans-Jürgen (Hrsg.): Aus gesamtwirtschaftlicher Sicht: Festschrift für Jürgen Kromphardt. Marburg: Metropolis: 19-29.

Rothschild, Kurt W. (2010): Die Kontroverse Frankfurter Allgemeine Zeitung contra Handelsblatt. Einige Bemerkungen zu einem methodologischen Schlagabtausch anno 2009. Intervention - Journal of Economics, 7(1): 24-31.

Samuelson, Paul A. (1973): Economics. 9. Auflage, McGraw-Hill.

Sent, Esther-Mirjam (2004): Behavioral Economics: How Psychology Made Its (Limited) Way Back Into Economics. History of Political Economy, Vol. 36(4): 735-760.

Stiglitz, Joseph E. (1988): On the Market for Principles of Economics Textbooks: Innovation and Product Differentiation. Journal of Economic Education, Vol. 19(2): 171-177.

Walpen, Bernhard (2004): Die offenen Feinde und ihre Gesellschaft. Eine hegemonietheoretische Studie zu Mont Pelerin Society. Hamburg: VSA.

Wilson, David, und Dixon, William (2009): Performing Economics: A Critique of 'Teaching and Learning'. International Review of Economic Education, Vol. 8(2): 91-105.

\section{"TRANSFORMATION IM KAPITALISMUS UND DARÜBER HINAUS» 1. INTERNATIONALE TRANSFRMMATIONSKONEREEDZ}

13. Oktober, 9:15 Uhr bis 14. Oktober 2011, 22:00 Uhr

\section{Auf der zweitägigen Konferenz sollen u.a. folgende Fragen diskutiert werden:}

Was heißt Transformation heute? Welche realen Alternativen stehen auf der Tagesordnung? Wie tief müssen die Veränderungen im Kapitalismus sein und inwieweit können und müssen sie über ihn hinausweisen?

Mit einer Einführung von Rainer Rilling zu:

Was heißt und zu welchem Ende betreiben wir sozialistische Transformationsforschung?

Zentrale Vorträge von Dieter Klein und Alex Demirovic zu Radikaler Realpolitik in Zeiten der Transformation. Mit anschließender Diskussion.

Vorträge und Kommentare u.a. von Frigga Haug, Ulrich Brand, Michael Brie, Radhika Desai, Thomas Seibert, Birgit Mahnkopf, Dieter Boris Anstelle eines Abschlusses: Beverly Silver: Jenseits des langen 20. Jahrhunderts
20:30 Uhr Ausklang: Konzert mit Gina Pietsch (mit Texten von Peter Hacks)

Anmeldung bitte bis zum 15. September 2011 Kontakt: Rosa-Luxemburg-Stiftung, Institut für Gesellschaftsanalyse: Uta Tackenberg, Tel. 030 44310-438, tackenberg@rosalux.de Ort: Münzbergsaal, Franz-Mehring-Platz 1, 10243 Berlin

Weitere Informationen unter: www.rosalux.de

ROSA LUXEMBURG STIFTUNG 